\title{
Anti-inflammatory effects of Gualou Guizhi decoction in transient focal cerebral ischemic brains
}

\author{
HAI XIA HU ${ }^{1,2}$, RU HUI LIN ${ }^{1,2}$, XIAO QIN ZHU ${ }^{1,2}$, ZUAN FANG LI ${ }^{1,2}$ and LI DIAN CHEN ${ }^{3}$ \\ ${ }^{1}$ Academy of Integrative Medicine; ${ }^{2}$ Fujian Key Laboratory of Integrative Medicine on Geriatrics; \\ ${ }^{3}$ College of Rehabilitation Medicine, Fujian University of Traditional Chinese Medicine, Fuzhou, Fujian 350108, P.R. China
}

Received December 31, 2014; Accepted February 20, 2015

DOI: $10.3892 / \mathrm{mmr} .2015 .3511$

\begin{abstract}
The aim of the present study was to explore the neuroprotective effects of Gualou Guizhi decoction (GLGZD) in a rat model of middle cerebral artery occlusion (MCAO). Sprague-Dawley rats were divided into three groups: Sham (no MCAO), MCAO (MCAO with no GLGZD treatment) and GLGZD (MCAO with GLGZD treatment). Rats in the MCAO and GLGZD groups were subjected to permanent occlusion of the left middle cerebral artery. Neurological function and infarct volume were measured. Microglial activation and inflammatory cell accumulation were measured using immunohistochemistry. mRNA and protein expression of inflammatory mediators were examined using reverse transcription-quantitative polymerase chain reaction and an enzyme-linked immunosorbent assay. The expression of proteins associated with the nuclear factor $\kappa-B$ $(\mathrm{NF}-\kappa \mathrm{B})$ inflammation signaling pathway was analyzed using western blotting. The results of the present study suggested that infarct size was significantly reduced and neurological behavior function was improved in rats with MCAO treated with GLGZD compared with rats in the MCAO group. Amoeboid microglial expansion and inflammatory cell migration were observed in the infarcted areas of rats in the GLGZD group and were not identified in those of the MCAO group. Target mRNA and protein levels, and inflammatory cell infiltration were significantly reduced in the GLGZD group compared with the MCAO model group. Notably, GLGZD treatment induced neuroprotective effects, reducing inflammation and inhibiting NF- $\kappa \mathrm{B}$ signaling
\end{abstract}

Correspondence to: Dr Li Dian Chen, College of Rehabilitation Medicine, Fujian University of Traditional Chinese Medicine, 1 Qiuyang Road, Fuzhou, Fujian 350108, P.R. China

E-mail: cld@fjtcm.edu.cn

Abbreviations: GLGZD, gualou guizhi decoction; MCAO, middle cerebral artery occlusion; TNF- $\alpha$, tumor necrosis factor- $\alpha$; IL-1 $\beta$, interleukin 1 $\beta$; MCP-1, monocyte chemotactic protein 1; Mac-2, macrophage galactose-specific lectin-2; MPO, myeloperoxidase; $\mathrm{NeuN}$, neuronal nuclei; NF- $\mathrm{B}$, nuclear factor $\kappa-\mathrm{B} ; \mathrm{I} \kappa \mathrm{B} \alpha$, inhibitor $\kappa \mathrm{B}-\alpha$; ANOVA, analysis of variance

Key words: gualou guizhi decoction, middle cerebral artery occlusion, cerebral infarction, inflammation, microglia, nuclear factor $\kappa-B$ compared with the MCAO group. Therefore, GLGZD may exhibit anti-inflammatory effects against ischemia-reperfusion brain injury and may be a therapeutic target for ischemic stroke.

\section{Introduction}

Stroke is the one of leading causes of morbidity and mortality worldwide $(1,2)$. Ischemic stroke is a sudden interruption of blood supply to the brain caused by the blockage of an artery, which may result in brain damage and neurologic dysfunction (3). The suppression of brain damage resulting from ischemic stroke is essential in order to prevent a decrease in the quality of life for patients. However, a therapeutic strategy for cerebral ischemia/reperfusion injury has yet to be established (4-6). Neuroinflammation following ischemia is characterized by the rapid activation of resident microglia and the infiltration of inflammatory cells. Neuroinflammation causes an increase in the expression of proinflammatory cytokines and reactive oxygen species, and may lead to blood-brain barrier disruption, brain edema, and cell necrosis and apoptosis. Recent studies have demonstrated that reactive microglia express inflammatory mediators that may increase the risk of stroke in patients with permanent middle cerebral artery occlusion (MCAO) and transient ischemia (7-9). The maintenance of microglial function during focal stroke may be more important than that of neurons. The mechanisms of microglial activation involve nuclear factor- $\kappa \mathrm{B}(\mathrm{NF}-\kappa \mathrm{B})$ and mitogen-activated protein kinases (MAPK) signaling pathways. The inflammatory responses to brain injury are associated with the pathogenesis of stroke (10-12). The selective inhibition of inflammatory cytokine activity is important for the development of effective treatments for brain ischemia and reperfusion injury (13).

Gualou Guizhi decoction (GLGZD), a traditional Chinese medicine, has been widely used in China for the treatment of stroke-induced spasticity $(14,15)$. A previous study has demonstrated that GLGZD treatment may inhibit spasticity resulting from ischemia by modulating glutamate levels in an experimental rat model (16). Furthermore, previous studies have suggested that GLGZD may exhibit anti-neuroinflammatory effects by suppressing microglial activation in a lipopolysaccharide (LPS)-induced microglial cell culture experimental model $(17,18)$. In light of the results of previous studies, it is hypothesized that GLGZD may be used for the treatment of cerebral ischemia injury via a number of pathological 
pathways. The present study investigated the underlying regulatory effects of GLGZD in an experimental rat model. In the present study, transient focal cerebral ischemia was induced in rats using a MCAO model and one group of the rats received GLGZD treatment. Inflammatory cytokine expression levels, microglial activation and neutrophil infiltration were measured, which are associated with the $\mathrm{NF}-\kappa \mathrm{B}$ signaling pathway and are indicative of a neuroinflammatory response. The aim of the present study was to investigate whether GLGZD exerted an anti-inflammatory and protective effect via the $\mathrm{NF}-\kappa \mathrm{B}$ signaling pathway, following cerebral ischemic injury.

\section{Materials and methods}

Animals. Male Sprague-Dawley rats (age, 6 weeks; weight, 200-250 g), were obtained from Shanghai SLAC Laboratory Animal Co., Ltd. (Shanghai, China). Rats were housed at constant temperature and relative humidity and exposed to a $12 \mathrm{~h}$ light and darkness cycle. They were treated according to the animal facility guidelines of Fujian University of Traditional Chinese Medicine (Fuzhou, China). The animals were fed standard rodent food and pure water, ad libitum. Experimental procedures were conducted strictly in accordance with international ethical guidelines and the National Institutes of Health Guide concerning the Care and Use of Laboratory Animals. This protocol was approved by the Institutional Animal Care and Use Committee of Fujian University of Traditional Chinese Medicine.

Preparation of herbal extracts. Medicinal plants were obtained from Guo Yi Tang Chinese Herbal Medicine Store (Fujian, China) for the preparation of the GLGZD extract. The preparation included a mixture of six crude plant extract ingredients: Trichosanthis radix, Ramulus cinnamomi, Paeonia lactiflora, Glycyrrhiza radix, Zingiber officinale Roscoe and Fructus jujubae in a ratio of $3: 3: 3: 2: 3: 3$. The mixture was incubated in double distilled water for $30 \mathrm{~min}$, and then heated to $100^{\circ} \mathrm{C}$ and refluxed twice for $2 \mathrm{~h}$. Subsequently, the mixture was filtered and concentrated using a rotary evaporator (RE-2000; Shanghai Yarong Biochemistry Instrument Factory, Shanghai, China) to a final concentration of $1.16 \mathrm{~g} / \mathrm{ml}(16,17)$.

Rat model and experimental grouping. The MCAO rat model was established according to the methods of a previous study (16). Briefly, the rats were anesthetized using 10\% chloral hydrate (Sinopharm Chemical Reagent Co., Ltd., Shanghai, China), subsequently the left common carotid artery (CCA), the left external carotid artery and the internal carotid artery (ICA) were isolated and exposed. A monofilament nylon suture coated with poly-L-lysine (Beijing Sunbio Biotech Co., Ltd., Beijing, China) was inserted though the CCA into the ICA $(\sim 8-20 \mathrm{~mm}$ beyond the carotid artery bifurcation) until resistance was felt. The neck incision was then closed. Blood flow to the brain was blocked for $2 \mathrm{~h}$ in order to induce ischemia, subsequently the suture was withdrawn slowly by $\sim 10 \mathrm{~mm}$ in order to permit perfusion. Rats that exhibited hemiparesis or an increase in body temperature were used for the experiments. Rats were then randomly divided into three groups ( $n=15$ per group): Sham group, rats received sham surgery (not MCAO); MCAO model group, rats were subjected to MCAO with no GLGZD treatment. GLGZD group, rats were subjected to MCAO and treated daily with GLGZD $(1.16 \mathrm{~g} / \mathrm{ml})$ for seven days.

Behavioral examination. Following $2 \mathrm{~h}$ and 7 days of perfusion, blinded observer evaluations of neurological deficits were conducted for eight rats per group. The criteria for the neurological severity score was graded from 0-4 (19): 0, rat movement without any neurological deficit; 1 , complete failure to move the right forepaw; 2, repeated circling to the right when crawling; 3 , falling to the right; 4 , complete loss of the ability to walk. Rats with score 0 or 4 were excluded from the subsequent stages of the investigation (20).

Assessment of cerebral infarct volume. Following the evaluation of neurological deficits, the rats were anesthetized using an intraperitoneal injection of $10 \%$ chloral hydrate $(100 \mathrm{~g} / 0.3 \mathrm{ml})$ and then decapitated. Brains were removed and placed on ice for isolation of the cerebral cortex, subsequently 2-mm coronal sections were prepared. The sections were stained using tetrazolium chloride (TTC; 20 g/l, Sigma-Aldrich, St. Louis, MO, USA) with phosphate-buffered saline (PBS), for $30 \mathrm{~min}$ at $37^{\circ} \mathrm{C}$, in order to measure brain cell death. TTC is converted into a red dye when taken up by living cells. Therefore, ischemic brain cells appeared white and non-ischemic brain cells appeared red. Images of the sections were captured using a digital camera (SX20; Canon Inc., Tokyo, Japan). Infarct volume was measured using Image analysis software (Image J 1.37, National Institutes of Health, Betheseda, MA, USA) and calculated as the percentage of infarcted volume of the total cortex volume.

Tissue collection. At the end of treatment, rats ( $\mathrm{n}=6$ per group) were sacrificed and brains were rapidly removed for TTC staining. Then, nine other rats in each group were anesthetized using an intraperitoneal injection of $10 \%$ chloral hydrate $(100 \mathrm{~g} / 0.3 \mathrm{ml})$ and then perfused transcardially with saline (250 $\mathrm{ml})$ and $4 \%$ paraformaldehyde $(250 \mathrm{ml})$, followed by rapid removal of the brain. The cortex was then dissected for immunohistochemistry, RNA isolation and protein extraction. Blood was collected via cardiac puncture using a heparinized syringe (Nanjing Chemical Reagent Co., Ltd., Nanjing, China) and centrifuged at $1,625 \mathrm{x}$ g for $20 \mathrm{~min}$ in order to obtain the plasma that was subsequently stored at $-80^{\circ} \mathrm{C}$.

Enzyme-immunosorbent assay (ELISA) cytokine analysis. Cytokine production in the plasma samples [tumor necrosis factor- $\alpha$ (TNF- $\alpha$ ), interleukin $1 \beta$ (IL-1 $\beta$ ), interleukin 6 (IL-6) and monocyte chemotactic protein 1 (MCP-1)] were measured using ELISA kits (R\&D Systems, Inc., Minneapolis, MN, USA), according to the manufacturer's instructions. Microwell absorbance was measured at $450 \mathrm{~nm}$ using a microplate reader (BioTek 8008, Bad Friedrichshall, Germany).

Hematoxylin \& eosin $(H \& E)$ staining. $H \& E$ histology was conducted in order to examine the histopathological alterations in ischemic brain samples. Brains were dissected and fixed in $4 \%$ paraformaldehyde at $4^{\circ} \mathrm{C}$ for $72 \mathrm{~h}$, and then dehydrated and embedded in paraffin blocks. Coronal sections (3-mm) were cut backward from the optic chiasma. Sections 
were deparaffinized and hydrated with decreasing concentrations of alcohol, stained with H\&E, and photographed under a microscope (DFC310 FX; Leica, Wetzlar, Germany).

Immunohistochemistry. Brains were dissected and fixed in $4 \%$ paraformaldehyde at room temperature. Tissue blocks were then dehydrated, embedded in paraffin and cut into 5- $\mu \mathrm{m}$ coronal sections. The paraffin sections were gently washed with PBS for 15 min followed by blocking with normal horse serum (containing 0.3\% $\mathrm{H}_{2} \mathrm{O}_{2}$; Santa Cruz Biotechnology, Inc., Dallas, TX, USA) in PBS for $30 \mathrm{~min}$. The sections were then incubated overnight at $4^{\circ} \mathrm{C}$ with anti-neuronal nuclei (anti-NeuN; cat. no. bs-1613R), anti-macrophage galactose-specific lectin-2 (anti-Mac2; cat. no. bs-9505R) and anti-myeloperoxidase (anti-MPO; cat. no. bs-4943R) primary monoclonal antibodies (Beijing Biosynthesis Biotechnology Co., Ltd., Beijing, China). Sections were then rinsed with $0.1 \%$ PBS Tween-20 ${ }^{\circledR}$ and incubated with a secondary antibody (antirabbit IgG; cat. no. SP-9001; Beijing ZSGB Biotechnology Co., Ltd., Beijing, China), for $1 \mathrm{~h}$ at room temperature. Subsequently, they were treated with a DAB peroxidase substrate kit (Maixin Bio, Fuzhou, China) at $4^{\circ} \mathrm{C}$ in order to visualize the immunoreaction. Brain sections were observed and photographed under a microscope, and the percentage of positively stained cells was measured using Image $\mathbf{J}$ software for a semi-quantitative evaluation.

Reverse transcription-quantitative polymerase chain reaction (RT-qPCR). RNA was isolated from ipsilateral cortical tissue $\left(\mathrm{n}=3\right.$ rats per group) using TRIzol ${ }^{\circledR}$ (Invitrogen Life Technologies, Carlsbad, CA, USA). RNA was then reverse-transcribed (PrimeScript $^{\mathrm{TM}}$ II 1st Strand cDNA Synthesis kit; Takara Bio, Inc., Otsu, Japan) from $2 \mu \mathrm{g}$ of total RNA, in order to generate cDNA, and amplified with an SYBR Green I quantitative PCR kit (Takara Bio, Inc.) using Applied Biosystems Prism 7500 (7500 software v2.0.5; Applied Biosystems Life Technologies, Carlsbad, CA, USA). Quantitative PCR was performed using the following primers (Takara Bio, Inc.): Forward: 5'-CACCACGCTCTTCTGTCTACTG-3' and reverse: 5'-GTACTTGGGCAGATTGACCTC-3' for TNF- $\alpha$; forward: 5'-GTAATGATCGTCAACGGGGGAGGAC-3' and reverse: 5'-CCAGCAAGCCTTGCAACCTTAACCTTAACCA-3' for IL-1 $\beta$; forward: 5'-CCACCACTACAGCAAGGG-3' and reverse: 5'-GAACTGGGCAGACTCAAA-3' for IL-6; forward: 5'-TCGGAACCAAATGAGATCAGAAC-3' and reverse: 5'-GAGGTGGTTGTGGAAAAGGTAGTG-3' for MCP-1; and forward: 5'-TGGAGTCTACTGGCGTCTT-3' and reverse: 5'-TGTCATATTTCTCGTGGTTCA-3' for GAPDH, which was used as an internal control. Results were normalized to GAPDH expression and the fold change in relative mRNA levels of the gene of interest was determined using the ${ }^{2-\Delta \Lambda} \mathrm{Ct}$ method.

Western blot analysis. Brain samples were dissected from the ipsilateral cortex and extractions were conducted using a lysis buffer (Beijing Solarbio Science \& Technology Co., Ltd., Beijing, China) containing the protease inhibitor phenylmethanesulfonylfluoride. Protein concentrations were determined using the bicinchoninic acid method Beijing Solarbio Science \& Technology Co., Ltd.). Samples $(50 \mu \mathrm{g})$ were denatured at $100^{\circ} \mathrm{C}$ for $5 \mathrm{~min}$ and separated by $10 \%$
SDS-PAGE. Proteins were then transferred to polyvinylidene fluoride membranes (EMD Millipore, Billerica, MA, USA), blocked with $5 \%$ non-fat milk and detected using the following primary antibodies: Mouse monoclonal p65 (cat. no. sc-8008), rabbit polyclonal phosphor-p65 (p-p65; cat. no. sc-33020), rabbit polyclonal inhibitor $\kappa \mathrm{B}-\alpha$ (ІкB- $\alpha$; cat. no. sc-847),

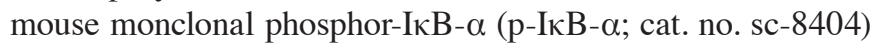
or goat polyclonal $\beta$-Actin (cat. no. sc-1616) $(1: 1,000)$ (Santa Cruz Biotechnology, Inc.) at $4^{\circ} \mathrm{C}$, overnight. Membranes were incubated with horseradish peroxidase-conjugated secondary antibodies (anti-rabbit IgG, cat. no. ZB-2301; and anti-mouse IgG, cat. no. ZB-2305; Beijing ZSGB Biotechnology Co., Ltd.) for $1 \mathrm{~h}$ at room temperature. Protein immunoblots were detected using enhanced chemiluminescence (RPN2132; GE Healthcare Bio-Sciences, Pittsburgh, PA, USA) for $1 \mathrm{~min}$, chemiluminescent bands were exposed to a Kodak film (Eastman Kodak, Rochester, NY, USA) in a dark room and the densitometry of the gel bands was measured using Image J software.

Statistical analysis. All results are represented as the mean \pm standard error of the mean. Data were analyzed using one-way analysis of variance using SPSS 15.0 (SPSS, Inc., Chicago, IL, USA). $\mathrm{P}<0.05$ was considered to indicate a statistically significant difference.

\section{Results}

GLGZD reduces cerebral infarction in rats. Brain infarct volume was determined in order to investigate the therapeutic effects of GLGZD on ischemic injury in rats that had experienced MCAO. According to the TTC staining, brain ischemia was not observed in rats in the sham group (Fig. 1). Infarct volume was significantly greater in rats in the MCAO group compared with those in the sham group. Infarct volume was significantly smaller in the GLGZD treatment group compared with those in the MCAO group (Fig. 1).

GLGZD treatment reduces neurological deficit in rats in the $M C A O$ group. In order to confirm the therapeutic effects of GLGZD on ischemia-induced spasticity, neurological deficit was examined. Rats in the MCAO group demonstrated motor functional disability, resulting in a higher neurological behavior score compared with the sham group (Fig. 2). GLGZD treatment led to a decrease in the neurological deficit score in rats following seven days of treatment. The results of the present study demonstrated that GLGZD treatment may reduce spasticity in rats following ischemic injury.

GLGZD suppresses inflammatory cytokine expression in rats in the MCAO group. Protein and mRNA expression levels of the following cytokines: TNF- $\alpha$, IL-1 $\beta$, IL- 6 and MCP-1, which are involved in neuroinflammation post-ischemia, were measured using ELISA and RT-qPCR. The results suggested that the cytokine expression levels were significantly greater in plasma samples from rats in the MCAO group, compared with those in the sham group. By contrast, GLGZD treatment exhibited significant inhibitory effects on cytokine expression compared with the MCAO group (Fig. 3A). These results were in accordance with the results of protein expression analyses. Cytokine mRNA levels were upregulated in rats in the MCAO 

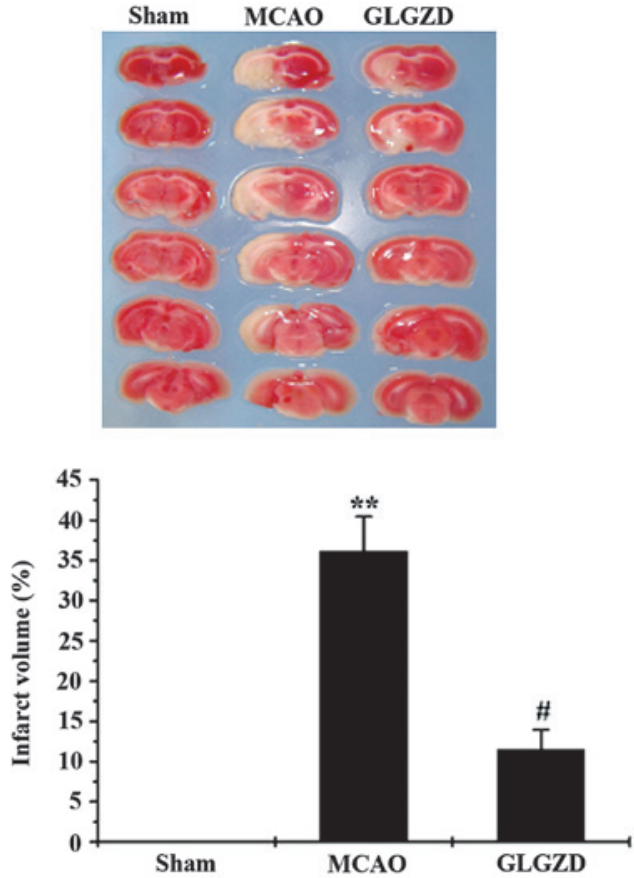

Figure 1. Effect of GLGZD treatment on cerebral infarction according to TTC staining. GLGZD was administered to rats in the GLGZD group for seven days following ischemia. Brain samples were then dissected and stained with TTC solution. Representative images of infarcted areas in coronal sections were visualized by TTC staining and infarct volumes are presented as the mean \pm standard error of the mean $(n=6){ }^{* * *} \mathrm{P}<0.01 \mathrm{vs}$. the sham group and ${ }^{~} \mathrm{P}<0.05$ vs. the MCAO group. GLGZD, Gualou Guizhi decoction; TTC, tetrazolium chloride; MCAO, middle cerebral artery occlusion.

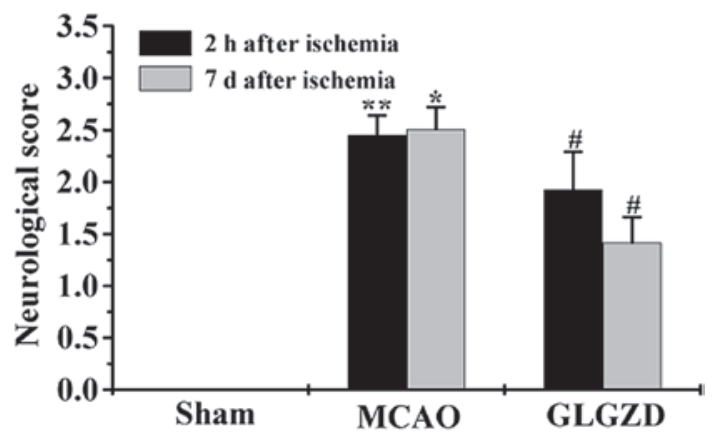

Figure 2. Effect of GLGZD on neurological deficits induced by MCAO using neurological deficit scores. The evaluation of neurological deficits was measured according to a four-point scale (0-4). Data are presented as the mean \pm standard errors of the mean $(n=5)$. ${ }^{*} \mathrm{P}<0.05$ and ${ }^{* *} \mathrm{P}<0.01$ vs. the sham group; ${ }^{\prime \prime} \mathrm{P}<0.05$ vs. the MCAO group. GLGZD, Gualou Guizhi decoction; $\mathrm{MCAO}$, middle cerebral artery occlusion.

group compared with those in the sham group, and GLGZD treatment led to significantly lower cytokine mRNA expression levels compared with the MCAO group (Fig. 3B).

GLGZD attenuates the inflammatory reaction and neuronal injury in rats in the MCAO group. Microglial activation, neutrophil infiltration and neuronal damage in the brain tissues were evaluated by analyzing cerebral histology using $\mathrm{H} \& \mathrm{E}$ and immunohistochemical staining with anti-NeuN, anti-Mac-2 and anti-MPO antibodies. H\&E and NeuN staining were performed in order to detect neuronal loss and nuclear
A
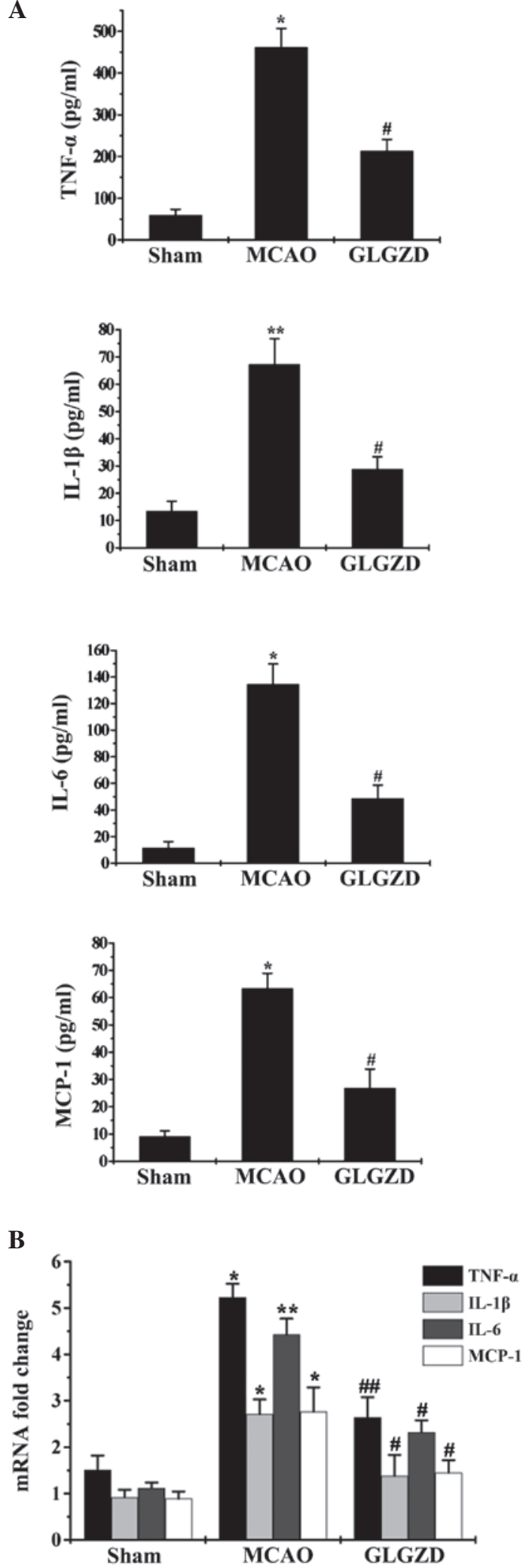

Figure 3. Effects of GLGZD treatment on cytokine protein and mRNA expression. (A) TNF- $\alpha$, IL-1 $\beta$, IL- 6 and MCP-1 production in peripheral blood was quantified using enzyme-linked immunosorbent assay. (B) Induction of mRNA expression in the cerebral cortex was assessed using reverse transcription-quantitative polymerase chain reaction. Quantitative data are represented as the mean \pm standard error of the mean of at least three independent experiments. ${ }^{*} \mathrm{P}<0.05$ and ${ }^{* *} \mathrm{P}<0.01$ vs. the sham group, and ${ }^{\#} \mathrm{P}<0.05$ and ${ }^{\# \#} \mathrm{P}<0.01$ vs. the MCAO group. GLGZD, Gualou Guizhi decoction; MCAO, middle cerebral artery occlusion; TNF- $\alpha$, tumor necrosis factor- $\alpha$; IL-1 $\beta$, interleukin $1 \beta$; IL-6, interleukin-6; MCP-1, monocyte chemotactic protein 1 . 
A

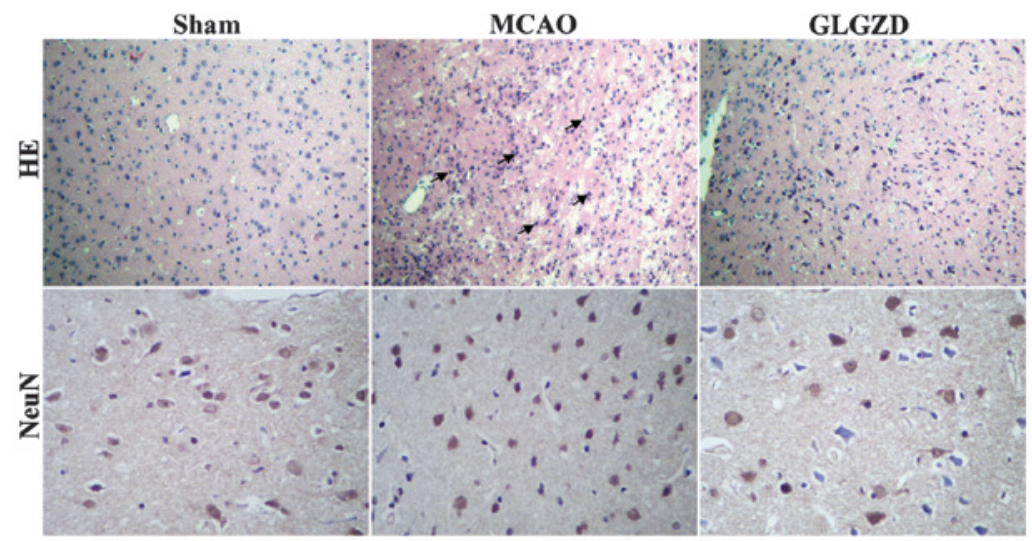

B

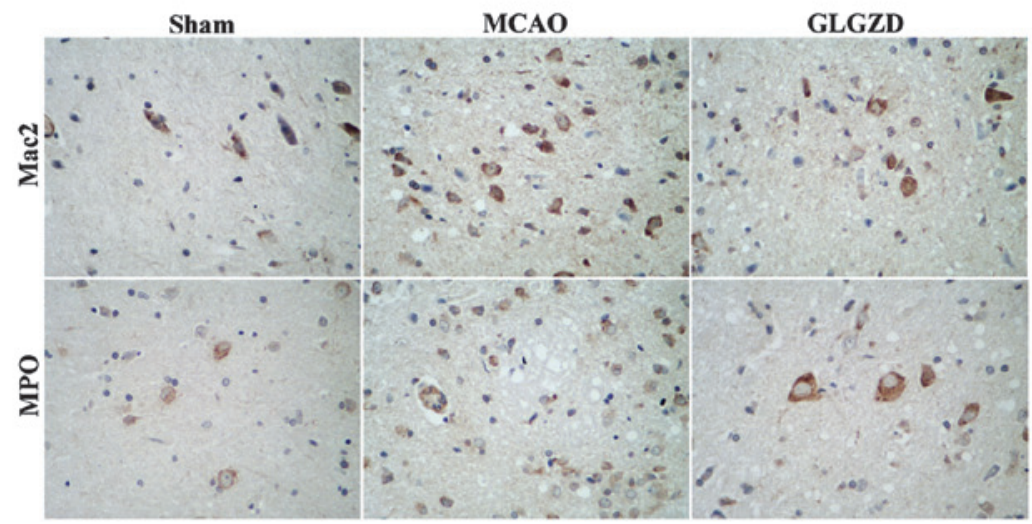

C

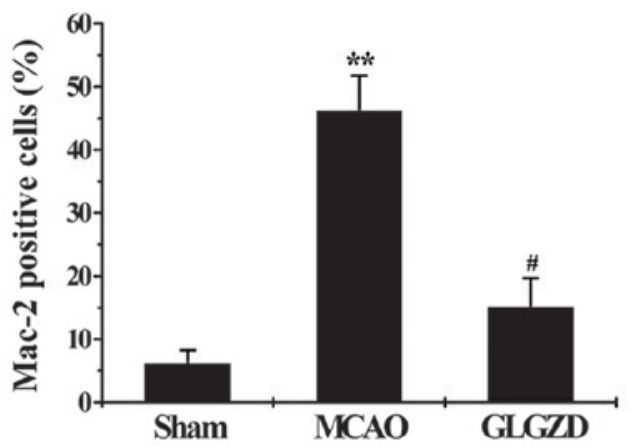

Figure 4. Effects of GLGZD treatment on neuronal damage and inflammatory responses in the ischemic contex were evaluated using H\&E staining and immunohistological analysis. Histopathological changes were measured using (A) H\&E staining (x200 magnification) (black arrows indicate nuclear shrinkage) and NeuN immunostaining, and (B) Mac2 and MPO immunostaining were performed in order to visualize the neurons, activated microglia and neutrophils, (x400 magnification). (C) Quantitative analyses of Mac2 and MPO are provided. Data are presented as the mean \pm standard error of the mean of five randomly selected microscope fields. ${ }^{*} \mathrm{P}<0.05$ and ${ }^{* *} \mathrm{P}<0.01$ vs. the sham group and ${ }^{\#} \mathrm{P}<0.05$ vs. the MCAO group. GLGZD, Gualou Guizhi decoction; MCAO, middle cerebral artery occlusion; H\&E, hematoxylin \& eosin; Mac-2, macrophage galactose-specific lectin-2; MPO, myeloperoxidase; NeuN, neuronal nuclei.

shrinkage. According to H\&E staining, the nuclei of neurons in the cerebral hemisphere of rats in the sham group were healthy, round and aligned. By contrast, the nuclei of neurons in the MCAO model group were pyknotic and few healthy neurons were observed in the core ischemic zone. Furthermore, the results of NeuN staining demonstrated marked neuronal shrinkage and reduction in rats in the MCAO group compared with the sham group. By contrast, GLGZD treatment led to lower levels of neuron death compared with the MCAO group (Fig. 4A). In order to demonstrate the anti-inflammatory effects of GLGZD treatment in rats that underwent MCAO, representative images of Mac-2 (an indicator of activated microglia) and MPO (an indicator of neutrophil infiltration) are shown in Fig. 4B. Low levels of activated microglia and neutrophil infiltration were observed in the cerebral cortex of rats in the sham group, compared with those in the MCAO group, as demonstrated by MPO and Mac-2. By contrast, lower levels of microglial activation and neutrophil infiltration were observed in the cerebral cortex of MCAO rats in the GLGZD group compared with those in the MCAO group (Fig. 4C).

GLGZD induces the reduction of inflammatory mediators associated with $N F-\kappa B$ signaling in rats in the MCAO group. In order to further investigate whether $N F-\kappa B$ signaling is 
$\mathbf{A}$

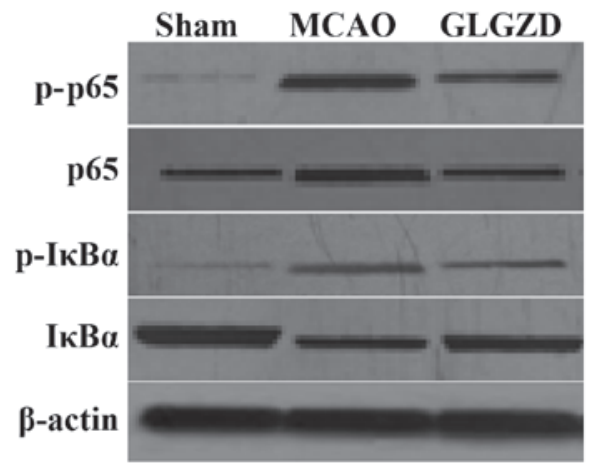

B
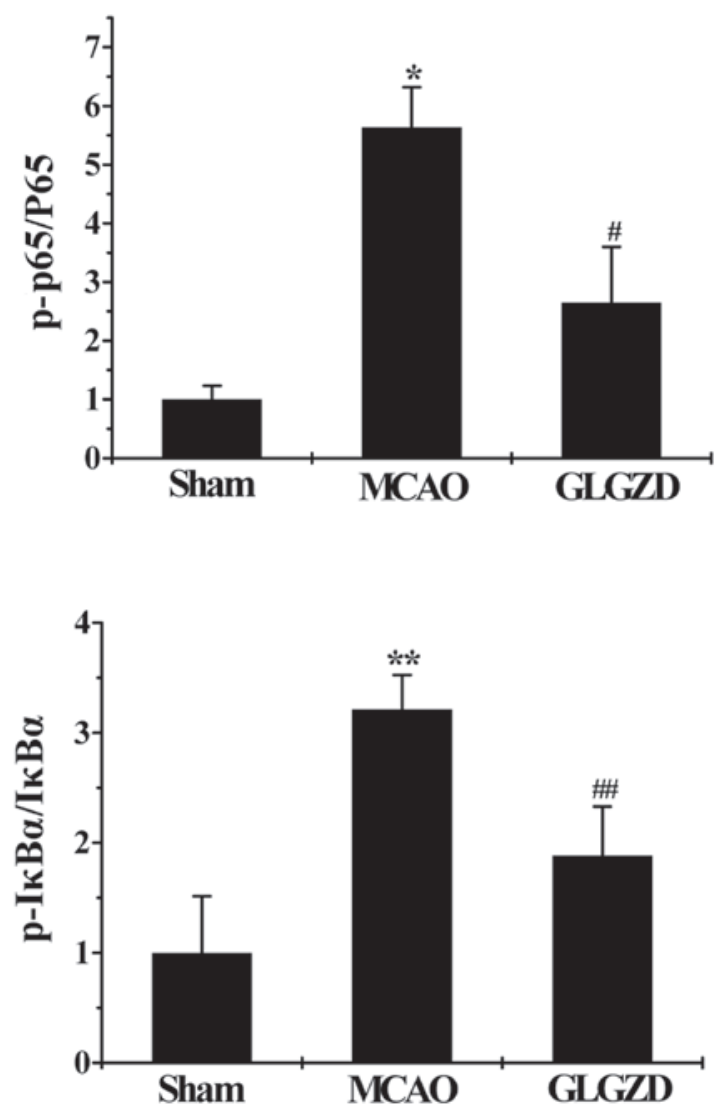

Figure 5. Effect of GLGZD treatment on the expression and phosphorylation of proteins associated with nuclear factor $\kappa$-B signaling. p65, phosphorylated $\mathrm{p} 65, \mathrm{IKB} \alpha$ and phosphorylated I $\mathrm{KB} \alpha$ expression levels were determined using western blot analysis. $\beta$-actin was used as an endogenous control. (A) Representative photographs are presented. (B) Denstitometric data are expressed as the mean \pm standard error of the mean. ${ }^{*} \mathrm{P}<0.05$ and ${ }^{* * *} \mathrm{P}<0.01$ vs. the sham group and ${ }^{\#} \mathrm{P}<0.05$ and ${ }^{\# \#} \mathrm{P}<0.01$ vs. the MCAO group. GLGZD, Gualou Guizhi decoction; MCAO, middle cerebral artery occlusion; p-p65, phosphor-p65; p-IкB $\alpha$, phosphor-inhibitor $\kappa \mathrm{B}-\alpha$.

associated with the anti-inflammatory effects of GLGZD, western blot analyses were performed. p-p65 expression was lower in the sham group compared with the MCAO group, and was significantly higher in the MCAO group compared with the GLGZD group. As shown in Fig. 5, IкBa phosphorylation was significantly higher in the MCAO group compared with the sham group, whereas treatment with GLGZD resulted in a decrease in IкBa phosphorylation, compared with the MCAO group. Furthermore, I $\mathrm{I} \mathrm{B} \alpha$ degradation was markedly blocked through suppressing the phosphorylated forms of I $\mathrm{B} \alpha$ in rats following treatment with GLGZD.

\section{Discussion}

Previous studies have demonstrated that the duration of ischemic stroke is associated with motor function disorders, such as spasticity $(21,22)$. A number of pathological events are associated with brain damage, including ischemic injury-induced neuroinflammation, which leads to tissue damage as indicated by the results of the present study. Microglia are the resident innate immune cells of the central nervous system (CNS) (23) and they are involved in host defense of the CNS. Inflammation in ischemic stroke is characterized by the rapid activation of resident microglia and the infiltration of inflammatory cells, including $\mathrm{MPO}^{+}$neutrophils and leukocytes. Following cerebral ischemia, neutrophils invade the cerebral parenchyma through the brain endothelium, and subsequently induce the inflammatory process. A number of inflammatory mediators, such as TNF- $\alpha$, IL- $1 \beta$, IL- 6 and MCP-1, which are indicators of neuroinflammation, are secreted by activated microglia (24). Inflammatory responses are associated with the NF- $\mathrm{KB}$ signaling pathway activation, which is involved in microglial activation (25). Upon NF- $\mathrm{kB}$ signaling activation, I $\mathrm{B} \alpha$ phosphorylation leads to I $\kappa \mathrm{B} \alpha$ degradation, causing $\mathrm{NF}-\kappa \mathrm{B}$ to translocate from the cytoplasm to the nucleus, resulting in the expression of the target genes (26). A number of studies have reported that the inhibition of inflammatory processes, for example via the production of inflammatory mediators, may reduce infarct area in MCAO models $(27,28)$.

GLGZD is a traditional Chinese medicine, consisting of a combination of six herbs, including Trichosanthis radix, Ramulus cinnamomi, Paeonia lactiflora, Glycyrrhiza radix, Zingiber officinale Roscoe and Fructus jujubae. GLGZD, an alternative therapy that may complement conventional medicine, has long been used in China to clinically treat post-stroke disabilities, such as muscular spasticity $(14,15)$. Studies have demonstrated that GLGZD treatment may contribute to the anti-inflammatory effects in LPS-induced microglial activation. To the best of our knowledge, the underlying mechanisms involved in of GLGZD neuroprotection are yet to be elucidated. Based on previous in vitro results, it is hypothesized that GLGZD may protect the brain from further neuronal damage in vivo by inhibiting microglial activation and inflammatory action $(17,18)$.

In the present study, an MCAO model was established in order to measure the anti-inflammatory effects of GLGZD on cerebral infarction and neurological deficit in rats with cerebral ischemia (29).

The effects of seven days of GLGZD treatment on ischemic-induced infarction and the level of neurological deficit in rats was investigated. GLGZD treatment significantly reduced the infarction volume and improved the neurological function in rats compared with rats without GLGZD treatment. Transcriptional and translational levels of inflammatory cytokines were measured using RT-qPCR and ELISA. Seven days of GLGZD treatment resulted in a reduction in the expression of neuroinflammation-associated mediators compared with the MCAO group. Cytokine and chemokine expression levels are associated with inflammatory cascade signaling, such as the 
$\mathrm{NF}-\kappa \mathrm{B}$ signaling pathway. GLGZD treatment led to a reduction in p-p65 (NF- $\kappa \mathrm{B}$ subunit) and p-I $\kappa \mathrm{B} \alpha$ expression levels, and an increase in p65 expression compared with the MCAO group.

In order to further examine neuronal morphologic changes and inflammatory responses in ischemic brain samples, neuron morphology, microglia activation and neutrophil infiltration were observed, using H\&E and immunohistochemical staining for NeuN, Mac-2 and MPO, seven days after treatment. Neuron loss and injury were lower in the GLGZD group compared with the MCAO group. In addition, the activation of Mac-2 and MPO observed in rats in the MCAO group was inhibited following GLGZD treatment, which suggests that GLGZD may exhibit an inhibitory effect on microglia activation and neutrophil infiltration.

In conclusion, the results of the present study demonstrate that GLGZD exhibits a therapeutic effect on ischemic injury in rats. Molecular mechanisms underlying these effects include the reduction of cytokine expression and inactivation of $\mathrm{NF}-\kappa \mathrm{B}$ signaling pathway. The present study provides novel insights into the molecular mechanisms underlying the neuroprotective effects of GLGZD and its potential as a novel therapeutic target for ischemic stroke.

\section{Acknowledgements}

The present study was supported by Natural Science Foundation of China (grant no. 81403265), The Guidance Project of the Fujian Provincial Department of Science \& Technology (grant no. 2012D012) and the Key Project of Department of Health of Fujian Province (grant no. zlckf01).

\section{References}

1. Lloyd-Jones D, Adams R, Carnethon M, De Simone G, Ferguson TB, Flegal K, Ford E, Furie K, Go A, Greenlund K, et al; American Heart Association Statistics Committee and Stroke Statistics Subcommittee: Heart disease and stroke statistics - 2009 update: A report from the American heart association statistics committee and stroke statistics subcommittee. Circulation 119: e21-e181, 2009.

2. Durai Pandian J, Padma V, Vijaya P, Sylaja PN and Murthy JM: Stroke and thrombolysis in developing countries. Int J Stroke 2: 17-26, 2007.

3. Sims NR and Muyderman H: Mitochondria, oxidative metabolism and cell death in stroke. Biochim Biophys Acta 1802: 80-91, 2010

4. Hishida R, Kamatani D, Kitaura H, Kudoh M and Shibuki K: Functionallocal connections with differential activity-dependence and critical periods surrounding the primary auditory cortex in rat cerebral slices. Neuroimage 34: 679-693, 2007.

5. Ginsberg LD: Impact of drug tolerability on the selection of antidepressant treatment in patients with major depressive disorder. CNS Spectr 14 (Suppl 12): 8-14, 2009.

6. Tuma RF and Steffens S: Targeting the endocannabinod system to limit myocardial and cerebral ischemic and reperfusion injury. Curr Pharm Biotechnol 13: 46-58, 2012.

7. Kim HJ and Chuang DM: HDAC inhibitors mitigate ischemia-induced oligodendrocyte damage: Potential roles of oligodendrogenesis, VEGF, and anti-inflammation. Am J Transl Res 6: 206-223, 2014.

8. Xie L, Sun F, Wang J, Mao X, Xie L, Yang SH, Su DM, Simpkins JW, Greenberg DA and Jin K: mTOR signaling inhibition modulates macrophage/microglia-mediated neuroinflammation and secondary injury via regulatory $\mathrm{T}$ cells after focal ischemia. J Immunol 192: 6009-6019, 2014.

9. Sheridan GK and Murphy KJ: Neuron-glia crosstalk in health and disease: Fractalkine and CX3CR1 take centre stage. Open Biol 3: 130181, 2013.
10. Vartanian KB, Stevens SL, Marsh BJ, Williams-Karnesky R, Lessov NS and Stenzel-Poore MP: LPS preconditioning redirects TLR signaling following stroke: TRIF-IRF3 plays a seminal role in mediating tolerance to ischemic injury. J Neuroinflammation 8: 140, 2011.

11. Sladojevic N, Stamatovic SM, Keep RF, Grailer JJ, Sarma JV, Ward PA and Andjelkovic AV: Inhibition of junctional adhesion molecule-A/LFA interaction attenuates leukocyte trafficking and inflammation in brain ischemia/reperfusion injury. Neurobiol Dis 67: 57-70, 2014.

12. Chen S, Yin ZJ, Jiang C, Ma ZQ, Fu Q, Qu R and Ma SP. Asiaticoside attenuates memory impairment induced by transient cerebral ischemia-reperfusion in mice through anti-inflammatory mechanism. Pharmacol Biochem Behav 122: 7-15, 2014.

13. Liu Y, Lian Z, Zhu H, Wang Y, Yu S, Chen T, Qu J, Li J, Ma S and Chen X: A systematic, integrated study on the neuroprotective effects of hydroxysafflor yellow A revealed by (1)H NMR-based metabonomics and the NF- $\kappa \mathrm{B}$ pathway. Evid Based Complement Alternat Med 2013: 147362, 2013.

14. Zhang L and Ai H: Effects of Gua Lou Gui Zhi decoction on c-fos and c-jun on epileptic rats. Sichuan J Tradit Chin Med 23: 21-22, 2005 (In Chinese).

15. Yang C, Chen L and Tao J: New usage of a classical formula-Gua Lou Gui Zhi decoction. Liaoning J Tradit Chin Med 39: 1599-1600, 2012 (In Chinese).

16. Huang J, Tao J, Xue X, Yang S, Han P, Lin Z, Xu W, Lin J, Peng J and Chen L: Gua Lou Gui Zhi decoction exerts neuroprotective effects on post-stroke spasticity via the modulation of glutamate levels and AMPA receptor expression. Int J Mol Med 31: 841-848, 2013.

17. Hu H, Li Z, Zhu X, Lin R, Lin J, Peng J, Tao J and Chen L: Gua Lou Gui Zhi decoction suppresses LPS-induced activation of the TLR4/NF- $\kappa \mathrm{B}$ pathway in BV-2 murine microglial cells. Int J Mol Med 31: 1327-1332, 2013.

18. Hu H, Li Z, Zhu X, Lin R, Peng J, Tao J and Chen L: GuaLou GuiZhi decoction inhibits LPS-induced microglial cell motility through the MAPK signaling pathway. Int J Mol Med 32: 1281-1286, 2013.

19. Xue X, You Y, Tao J, Ye X, Huang J, Yang S, Lin Z, Hong Z, Peng $J$ and Chen L: Electro-acupuncture at points of Zusanli and Quchi exerts anti-apoptotic effect through the modulation of PI3K/Akt signaling pathway. Neurosci Lett 558: 14-19, 2014.

20. Longa EZ, Weinstein PR, Carlson S and Cummins R: Reversible middle cerebral artery occlusion without craniectomy in rats. Stroke 20: 84-91, 1989.

21. Jiang B, Wang WZ, Chen H, Hong Z, Yang QD, Wu SP, Du XL and Bao QJ: Incidence and trends of stroke and its subtypes in China: Results from three large cities. Stroke 37: 63-68, 2006.

22. Guo JM, Liu AJ and Su DF: Genetics of stroke. Acta Pharmacol Sin 31: 1055-1064, 2010.

23. Zhang L, Dong LY, Li YJ, Hong Z and Wei WS: The microRNA miR-181c controls microglia-mediated neuronal apoptosis by suppressing tumor necrosis factor. J Neuroinflammation 9: 211, 2012.

24. Lu X, Ma L, Ruan L, Kong Y, Mou H, Zhang Z, Wang Z, Wang JM and Le Y: Resveratrol differentially modulates inflammatory responses of microglia and astrocytes. J Neuroinflammation 7: 46, 2010.

25. Kim JB, Yu YM, Kim SW and Lee JK: Anti-inflammatory mechanism is involved in ethyl pyruvate-mediated efficacious neuroprotection in the postischemic brain. Brain Res 1060: 188-192, 2005.

26. Perkins ND: Integrating cell-signalling pathways with NF-kappaB and IKK function. Nat Rev Mol Cell Biol 8: 49-62, 2007.

27. Guo RB, Wang GF, Zhao AP, Gu J, Sun XL and $\mathrm{Hu}$ G: Paeoniflorin protects against ischemia-induced brain damages in rats via inhibiting MAPKs/NF- $\kappa$ B-mediated inflammatory responses. PLoS One 7: e49701, 2012.

28. Dejda A, Seaborn T, Bourgault S, Touzani O, Fournier A, Vaudry H and Vaudry D: PACAP and a novel stable analog protect rat brain from ischemia: Insight into the mechanisms of action. Peptides 32: 1207-1216, 2011.

29. Gibson CL: Cerebral ischemic stroke: Is gender important? J Cereb Blood Flow Metab 33: 1355-1361, 2013. 